\title{
L'intégration de la recherche à la médecine clinique : difficultés et solutions en contexte canadien
}

\author{
François Lamontagne MD MSc, Kathryn M. Rowan PhD, Gordon Guyatt MD OC
}

- Citation: CMAJ 2021 January 25;193:E127-31. doi : 10.1503/cmaj.202397-f; diffusion hâtive le 9 décembre 2020

Voir la version anglaise de l'article ici : www.cmaj.ca/lookup/doi/10.1503/cmaj.202397

B ien que le Canada ait investi des centaines de millions de dollars dans la recherche sur la maladie à coronavirus 2019 (COVID-19), la productivité d'autres pays en la matière a largement dépassé la nôtre. Prenons l'exemple du Royaume-Uni : avec l'infrastructure de son Clinical Research Network, le National Institute for Health Research (NIHR) a fait l'envie du monde entier, lui qui a su enclencher rapidement des travaux de recherche et diffuser des connaissances dans les mois suivant le début de la pandémie, ce qui a poussé certaines personnes à réclamer que le Canada imite le système britannique ${ }^{1}$. On a utilisé davantage de fonds de recherche au pays pendant la pandémie, mais il en faudrait possiblement plus. Cela dit, l'argent ne suffira pas pour exploiter le plein potentiel du secteur canadien de la recherche en santé; un changement de culture s'impose, tout comme l'établissement d'un partenariat entre les systèmes de santé des provinces et des territoires et les nombreux instituts et organismes de recherche.

Dans cet article, nous discutons des limites de l'infrastructure de recherche clinique au Canada, décrivons les mécanismes qui ont permis l'intégration de ce type de recherche dans le système de santé du Royaume-Uni et proposons un mode d'emploi pour la création d'une version canadienne du système britannique en mentionnant les pièges à éviter.

\section{Qu'est-ce qui nuit à la recherche clinique au Canada?}

Trois grands problèmes alourdissent le déroulement de la recherche au Canada. Si personne ne les règle, toute tentative de modernisation de l'infrastructure de recherche en santé va vraisemblablement échouer.

\section{L'infrastructure de recherche est inefficace}

La réalisation d'études cliniques (essais et études d'observation prospectives) demande le soutien d'une infrastructure de recherche. Quand cette infrastructure n'existe pas déjà, les chercheurs doivent la créer dans chaque établissement participant avant d'entamer leur projet, ce qui n'est pas une mince affaire. Pour la plupart des études cliniques, la planification et l'exécution de

\section{POINTS CLÉS}

- Malgré les centaines de millions de dollars investis dans la recherche sur la maladie à coronavirus 2019 (COVID-19) au Canada, la productivité d'autres pays en la matière a largement dépassé la nôtre.

- Au Royaume-Uni, le National Institute for Health Research a su, grâce à l'infrastructure de son Clinical Research Network, enclencher rapidement des travaux de recherche et diffuser des connaissances sans tarder. Le Canada pourrait imiter le système britannique.

- Parmi les problèmes qui nuisent à l'efficience et à la productivité de la recherche clinique au Canada, on compte l'inefficacité de l'infrastructure de recherche, la nonconcertation des efforts et la culture voulant que la recherche soit séparée de la médecine clinique.

- Pour favoriser un changement dans la culture de la recherche médicale au Canada et établir un partenariat entre les systèmes de santé et les instituts et organismes de recherche, il faut une volonté politique tant au fédéral qu'au provincial.

- Il faut plusieurs éléments pour enclencher le processus : une analyse minutieuse des coûts et des problèmes d'efficience du système actuel, des projections réfléchies du rendement du financement alloué à une infrastructure plus efficace, et des administrations provinciales ou territoriales désireuses de collaborer à l'essai d'un nouveau modèle.

cette démarche font intervenir de nombreux acteurs (services des urgences et services de soins intensifs des hôpitaux, consultants clés, pharmacies, banques de sang, administrations, syndicats, etc.). La mise en place d'infrastructures pour chaque étude est au mieux inefficace et coûteuse. Dans le pire des cas, l'insuffisance des ressources, de l'expérience ou de l'expertise pour la création d'infrastructures dans un établissement candidat en particulier enlève aux Canadiennes et Canadiens de précieuses occasions de participer à d'importants travaux de recherche en santé, paralyse le développement d'une expertise chez les scientifiques du pays et empêche les systèmes locaux de produire des données qui vont éclairer les décisions cliniques 
individuelles et systémiques. Les leaders des Instituts de recherche en santé du Canada (IRSC) ne sont pas nécessairement au courant de ces coûts récurrents ni de la quantité de fonds engloutis dans des projets en santé qui n'aboutissent pas, faute de capacités à créer l'infrastructure nécessaire.

\section{La recherche est vue comme une culture à part}

Actuellement, les travailleurs de la santé (personnel infirmier, médecins, professionnels paramédicaux, personnel auxiliaire, administrateurs, etc.) jouent pour la plupart un rôle périphérique dans les études cliniques, ou ne jouent pas de rôle du tout. La responsabilité de la recherche revient presque exclusivement à un petit groupe de personnes dévouées qui œuvrent dans un écosystème fragile.

Il n'est pas rare que les travailleurs de la santé estiment que les chercheurs ne font pas partie de leur clan, de la "véritable main-d'œuvre en santé ». En même temps, les professionnels de la santé clinique ont peu d'influence sur les sujets étudiés, ce qui creuse le fossé entre la recherche et la pratique.

Les fonds alloués à la recherche sont souvent vus comme des sommes faramineuses qui auraient dû aller aux services de santé, ce qui vient accentuer le problème et accroître la réticence des établissements à s'engager dans des études cliniques. Et pour ceux qui font le saut, leur contribution est parfois limitée (p. ex., petit nombre de participants ou recrutement lent, nécessitant souvent un financement prolongé), et le respect du protocole, lacunaire 2 .

Or, comme l'optimisation d'un système de santé public repose sur l'évaluation continue et minutieuse de l'efficacité clinique et de l'efficience des interventions, il est absolument essentiel de combler le fossé entre la recherche et la pratique cliniques. La pandémie de COVID-19 nous a montré ce qui se passe quand les décisions en santé ne sont pas fondées sur des données fiables: dans le meilleur scénario, les systèmes gaspillent des ressources déjà anémiques; dans le pire scénario, des patients souffrent et meurent. Tant que le système ne trouvera pas son compte dans la recherche, ce sont d'autres groupes d'intérêt qui vont dicter les programmes en la matière.

\section{Les efforts ne sont pas concertés}

Des médias ont qualifié la recherche sur la COVID-19 au Canada d'éparpillée et de chaotique ${ }^{3}$. L'absence de coordination bloque toute avancée, même si tout le reste fonctionne. Par exemple, il n'existe aucun mécanisme qui empêcherait les comités des IRSC de subventionner des projets concurrents... sans compter que les IRSC ne sont pas le seul organe de financement de la recherche clinique au pays. Qu'on parle de la grosse enveloppe des IRSC ou encore des petits montants octroyés par des organismes à but non lucratif, des services d'hôpitaux et des centres de recherche, les bailleurs de fonds continuent à lancer sans se consulter des initiatives séparées visant à répondre à des questions similaires, ce qui, compte tenu des ressources limitées, est absolument inquiétant. Le chevauchement dans l'allocation du financement et les doublons de projets de recherche traduisent la possibilité ratée d'unifier la communauté scientifique canadienne autour d'objectifs communs et de se pencher sur les brèches dans les connaissances, qui, sans planification stratégique, ne seront jamais colmatées.

\section{Quel système de recherche national serait considéré comme excellent?}

Un éditorial récemment publié dans le CMAJ souligne l'impressionnant tour de force réalisé par l'équipe de l'essai Randomised Evaluation of COVID-19 Therapy (RECOVERY), au RoyaumeUni, et sa contribution massive et rapide aux connaissances sur les effets des interventions contre la COVID-19 ${ }^{1}$. L'essai RECOVERY a utilisé l'infrastructure du Clinical Research Network, associé au NIHR. Le modèle du NIHR, qui met à profit le réseau de recherche clinique financé à long terme préexistant, lequel fait partie intégrante et reçoit du financement du National Health Service (NHS), a réglé simultanément les problèmes liés à la mise en place d'infrastructures ainsi qu'à la participation et à la fragmentation du système de santé, en contexte britannique. À l'arrivée de la pandémie, grâce à la flexibilité et à la capacité d'adaptation de son infrastructure, les équipes d'essais en cours tels que le Randomised, Embedded, Multi-factorial, Adaptive Platform trial for Community-Acquired Pneumonia (REMAP-CAP) ont pu faire appel à plus d'établissements au Royaume-Uni que dans tous les autres pays participants réunis (les établissements britanniques ont recruté $73 \%$ des patients atteints de la COVID-19 participant à REMAP-CAP) ${ }^{4}$. En outre, 94\% des données qui ont servi à l'élaboration des récentes orientations sur l'utilisation de corticostéroïdes pour le traitement de la COVID-19 par l'Organisation mondiale de la Santé provenaient de ce pays ${ }^{5}$.

Comme le Royaume-Uni et le Canada sont intrinsèquement différents, le modèle du NIHR devra être adapté au contexte canadien. L'adaptation devra cependant tenir compte des étapes ayant mené à la création du NIHR et des principaux ingrédients de son succès. L'Institut a un large mandat, comprenant la réalisation de recherche translationnelle dans ses premières phases, d'essais cliniques et de recherche appliquée en santé et en services de santé, et ce, dans de multiples programmes. Nous nous concentrons toutefois sur les éléments du modèle qui favorisent la tenue d'études cliniques comme celles décrites ci-dessus.

\section{Le résultat d'une volonté politique}

En 2006, le gouvernement britannique, conscient que le rendement de la recherche clinique était inférieur aux attentes, et après des années de profonde réflexion sur le rôle de la recherche dans les systèmes de santé ${ }^{6}$ a adopté une politique voulant que le réseau de la santé (NHS) soit l'un des principaux intervenants dans les activités de recherche dans le domaine ${ }^{7}$. Pour y arriver, alors que le financement en santé et le financement de la recherche en santé étaient séparés, les responsables du NHS ont investi dans les infrastructures nécessaires à la tenue d'études cliniques dans l'ensemble des centres de santé du pays, notamment en finançant des changements structurels (qui ne seront pas décrits dans cet article, mais le sont ailleurs ${ }^{8,9}$ ). Il est important de comprendre que c'est la classe politique, et non le milieu scientifique, qui a voulu ces changements.

\section{Un financement intelligent des infrastructures, pour une participation accrue}

Fondamentalement, le NHS a réorienté des fonds " qui dormaient » dans les organismes de santé, qui étaient destinés à leur mission scientifique (donc pas alloués selon les besoins, mais plutôt selon 
leurs affectations habituelles ${ }^{7}$ ). Cette réorientation graduelle a soutenu l'établissement et le maintien d'un réseau de recherche clinique rassemblant du personnel, des installations, des équipements et des services de soutien réservés à l'exécution d'études cliniques, à maints endroits. Le financement total n'a pas changé, mais le fait de ne plus avoir à créer d'infrastructures pour chaque étude clinique locale a considérablement amélioré l'efficience économique.

Désormais, le système de santé investissait dans les études cliniques. Dans tous les systèmes, le payeur a beaucoup plus d'influence sur les parties en cause qu'une communauté scientifique non soudée. En fait, le NHS a commencé à exploiter cette influence dans le but que chaque composante du système fasse sa part pour assurer une prise de décision fondée sur des données probantes. Une fois le rendement de la recherche et d'autres critères d'allocation des fonds publics en vigueur, les perceptions ont changé : les professionnels de la santé et les administrateurs ont rapidement pris goût à la recherche de qualité, ce qui a métamorphosé la culture de la recherche ${ }^{8}$.

Les changements ont grandement contribué à concerter les efforts. En ayant à établir des règles et procédures claires concernant l'accès à la nouvelle infrastructure collective de recherche, les responsables ont dû tenir compte de la nécessité de maximiser l'efficience de la recherche. Ils ont atteint l'objectif en rassemblant les études jugées prioritaires par les comités de représentants, classées par domaines cliniques. Le NIHR surveille étroitement la progression de ces études dans chacun des domaines, et attend que l'une soit terminée avant d'ouvrir l'infrastructure de recherche clinique aux chercheurs d'un projet concurrent. Cette façon de faire prévient la fragmentation et la compétition improductive entre les études financées. Et le NIHR va plus loin : il a implanté des mécanismes qui empêchent les projets qui n'aboutissent pas de nuire à l'ensemble du système et qui encouragent la saine compétition entre les groupes de recherche. Puisqu'ils peuvent ne plus recevoir de fonds s'ils n'atteignent pas les objectifs fixés, les chercheurs autorisés à utiliser la précieuse infrastructure collective du Clinical Research Network se doivent de produire des résultats.

\section{Autres avantages indirects : diversification de la recherche, méritocratie et économies}

Par le passé, les groupes de recherche brandissaient souvent les réalisations de « leurs » infrastructures de recherche, concurrentes, pour obtenir du financement, perpétuant ainsi les modalités inefficaces d'attribution des subventions. Le nouveau modèle a forcé les scientifiques établis à partager la « propriété » de l'infrastructure de recherche clinique et à briser ce cycle improductif. II a également donné plus de pouvoir aux décideurs, aux responsables des orientations politiques, aux patients et au public sur la nature de la recherche. Le NIHR favorise maintenant les projets qui comptent pour la population britannique, mais encourage toujours les scientifiques à faire des propositions innovantes. Par conséquent, le centre de coordination du Clinical Research Network impose (fait valoir) certaines idées, et en accepte (reçoit) d'autres, de la part des chercheurs. La circulation d'idées dans les deux sens facilite l'intégration de projets moins rassembleurs qui autrement n'auraient probablement jamais reçu de financement, projets que l'on appelle parfois des « défaillances du marché ${ }^{10}$ ».
En présence d'une infrastructure de recherche à la fois collective et efficace, il est possible d'évaluer les projets proposés en fonction du modèle d'étude et de la portée de la question de recherche, et non de la capacité des chercheurs, par leur influence politique, à enrôler suffisamment d'établissements. Et si les acteurs en jeu jugent important de former les prochaines générations de scientifiques et de faire participer les organismes de santé, les gestionnaires, les professionnels de la santé, les patients et le public dans le processus, ils peuvent, pour y arriver, mettre à profit le potentiel de l'infrastructure nationale ${ }^{11}$.

Depuis 2006, la rigoureuse analyse comparative des coûts et des bénéfices montre que le NIHR produit un bon rapport qualité-prix. Des évaluations indépendantes du modèle britannique ont conclu « qu'entre 2016-2017 et 2018-2019, les activités de recherche clinique financées par le Clinical Research Network ont augmenté la valeur brute de la recherche d'environ 8 milliards de livres et ont créé 47467 postes à temps plein ». D'après la même source, l'infrastructure a attiré des investissements privés indispensables, entraînant par le fait même une diminution des coûts pour le système ${ }^{12}$. D'autres études ont montré que l'efficacité accrue des soins avait des avantages sur le plan économique. Le gouvernement du Royaume-Uni a donc choisi de renouveler le budget du NIHR chaque année.

D'après une étude de la RAND Corporation, « $12 \%$ du bénéfice net potentiel de l'application pendant 1 an des conclusions d'un échantillon de 10 études suffirait à couvrir les dépenses associées au Programme pour la période 1993-2012 ${ }^{13}$ ». Voilà un argument pour la réalisation d'évaluations économiques scrupuleuses des réseaux de recherche clinique et la considération des différentes façons dont la qualité des données produites peut engendrer des économies ${ }^{14}$.

\section{Comment mettre sur pied un réseau canadien de recherche clinique?}

\section{Première étape : Instaurer le dialogue}

L'adaptation du modèle du NIHR au contexte canadien demandera de la volonté politique, comme ce fut le cas au RoyaumeUni. À ce sujet, la pandémie a peut-être jeté les bases qui faciliteront l'amorce d'une conversation nécessaire. Pour parler de la possibilité d'apporter des changements à la structure de la recherche en santé au Canada, il faut que les gouvernements fédéral et provinciaux soient à la table. Heureusement, cette formule est déjà utilisée, lors des négociations annuelles des transferts en santé entre Ottawa et les provinces. De plus, comme le font les ministres fédéral, provinciaux et territoriaux de la Santé, les membres et les bailleurs de fonds de l'Alliance canadienne des organismes provinciaux de recherche en santé se rencontrent régulièrement. La création d'une infrastructure collective de recherche clinique serait, pour le gouvernement canadien, une occasion en or de contribuer à l'amélioration des systèmes de santé du pays par l'affectation d'une portion des transferts fédéraux à cette entreprise. Peut-être les provinces verraientelles l'interface de la santé et de la recherche comme un terrain neutre apprécié. Puisqu'historiquement, la recherche est de 
compétence fédérale, les provinces pourraient enfin utiliser l'infrastructure pour se pencher sur leurs propres priorités, ce qui améliorerait la recherche et ainsi créerait un système de santé revampé, plus efficient et viable.

\section{Deuxième étape : Faire des évaluations initiales et des analyses comparatives}

L'analyse exhaustive des sources de financement de la recherche clinique au Canada (IRSC, financement de la santé par les provinces, fondations privées, etc.) pourrait être le point de départ de discussions approfondies sur les modalités de financement. Même si dans l'absolu, les coûts d'un réseau collectif seraient substantiels, les fonds de recherche perdus dans différents projets de recherche non coordonnés à l'échelle du pays le sont tout autant; c'est pourquoi une évaluation juste des dépenses doit aussi estimer ce que coûte le statu quo. Il sera tout aussi capital, en parallèle, de faire une liste exhaustive des études cliniques coexistantes à l'échelle nationale, indiquant notamment la durée prévue, le lieu de recrutement des participants et la mesure dans laquelle les projets se battent pour les mêmes participants.

\section{Troisième étape : Évaluer le rendement des investissements}

La mise en pratique des résultats de recherche entraîne parfois l'abandon ou l'élimination d'interventions onéreuses pour des interventions moins couteuses aux avantages cliniques comparables. Comme dans l'exemple du Royaume-Uni, l'efficience fait économiser de l'argent. C'est pourquoi les provinces, en tant que responsables des systèmes de santé au Canada, ont beaucoup à gagner à investir dans un réseau.

Il sera donc crucial de planifier les méthodes de suivi et d'évaluation indépendante du rendement de la recherche au pays. Les détracteurs diront que la supériorité du modèle du NIHR n'est pas aussi nette en l'absence d'une crise sanitaire mondiale, mais la réalité est qu'il n'existe pas de manière idéale de mesurer ce rendement. L'utilisation d'indicateurs de processus pourrait aussi être importante. Par exemple, si l'élimination d'interventions inefficaces, la participation par personne des patients inclus dans les études cliniques, la durée des projets et le coût final des études vont tous dans le même sens (indiquent une amélioration), alors le maintien de la nouvelle infrastructure serait justifié.

\section{Quatrième étape : Avancer petit à petit, et éviter les pièges}

L'obtention d'un consensus national concernant l'imitation du système britannique a de fortes chances de prendre du temps, et le travail, de devoir se faire par étapes. Au début, quelques provinces volontaires pourraient réaliser un projet pilote, et d'autres suivront si l'expérience s'avère concluante. Avant l'arrivée de la COVID-19, plusieurs réseaux cliniques stratégiques ont vu le jour en Alberta, mais bien qu'on y promeuve la recherche en principe, le financement alloué à l'infrastructure de recherche demeure limité. Pendant la pandémie, le gouvernement du Québec a créé un réseau interdisciplinaire (le Réseau québécois COVID Pandémie) chargé d'explorer les différentes façons d'améliorer la coordination de la recherche entre les secteurs et les disciplines jusqu'à maintenant mal intégrés aux autres. Un de ses groupes de travail tient des rencontres de discussion spécialement sur les réseaux de recherche clinique ${ }^{15}$. Dans ce contexte, il se peut que des provinces, prenant conscience que les premiers utilisateurs auront probablement une plus grande influence sur le produit final, choisissent d'elles-mêmes de participer à la création d'un réseau. On peut penser à l'essai d'un système d'assurance public des services médicaux en Saskatchewan, qui a valu à Tommy Douglas le titre de père de l'assurance-maladie au Canada.

Une autre possibilité serait d'implanter un projet pilote dans l'ensemble des provinces et territoires, mais dans un seul domaine clinique. Voici un exemple de cette façon de faire aux États-Unis : le Collaborating Network of Networks for Evaluating COVID-19 and Therapeutic Strategies (CONNECTS), affilié au National Heart, Lung, and Blood Institute. Bien qu'il lui manque l'ingrédient actif qu'est l'intégration de la recherche dans un système de santé public, CONNECTS subventionne une infrastructure de coordination centrale et l'établissement de priorités. De plus, il profite de la cohésion des réseaux actuels, qui peut donner lieu à des économies d'échelle considérables ${ }^{16}$. C'est vraisemblablement ce qui a inspiré les IRSC à créer le Réseau des réseaux, pour le Canada. Malheureusement, CONNECTS n'a pour le moment pas prouvé grand-chose, par rapport aux énormes sommes d'argent investies dans la recherche sur la COVID-19 aux États-Unis. Pour obtenir de meilleurs résultats ici, il faudra mieux intégrer le réseau dans les systèmes de santé des provinces. Autrement dit, si le réseau choisit de ne pas entamer de discussions avec les deux ordres de gouvernement, il devra veiller à réserver des ressources aux établissements participants plutôt que de tout distribuer aux chercheurs.

Le modèle d'affaires actuel, selon lequel les ressources de recherche appartiennent aux chercheurs accomplis, amène invariablement ceux qui sont influents à faire des négociations préparatoires, c'est-à-dire à promouvoir, après avoir scellé une entente, leurs propres études. Ils se servent ensuite de leurs projets financés par la population, dans leurs demandes de subvention subséquentes, comme preuves de leur capacité à mettre sur pied l'infrastructure de recherche nécessaire pour leur prochain projet. Ce cercle est le symptôme d'un système déficient dans lequel le succès scientifique passe par le privilège, les relations politiques et l'argent, qui permettent d'exercer une influence sur les établissements participants. Il sera toujours important d'avoir une feuille de route jalonnée de succès; cependant, la création d'une infrastructure durable de recherche clinique laisserait les comités d'évaluation par les pairs accorder davantage de valeur à la question de recherche et au modèle d'étude.

\section{Conclusion}

L'infrastructure de recherche canadienne est inadéquate principalement parce que la recherche en santé et le domaine de la santé en tant que telle sont souvent vus comme des éléments séparés, qui devraient évoluer dans 2 mondes à part.

Il y a longtemps qu'on aurait dû parler des solutions pour remédier au déficit scientifique qui règne dans nos systèmes de santé et à ses effets. L'amélioration des services de santé est impossible sans prise de décision fondée sur des données probantes, et donc sans recherche clinique d'excellence qui répond aux besoins des systèmes. Notre part minime dans la recherche mondiale visant 
à trouver des traitements efficaces contre la COVID-19 et à discréditer les traitements inutiles et dangereux révèle un système déficient. En comparaison, le modèle du Royaume-Uni nous montre, sans l'ombre d'un doute, que la mise sur pied d'un réseau de recherche clinique qui serait collectif et financé par le gouvernement viendrait régler le problème. Le temps est venu de suivre l'exemple.

\section{Références}

1. Murthy S, Fowler RA, Laupacis A. How Canada can better embed randomized trials into clinical care: CMAJ 2020;192:E928-E29.

2. Kasenda B, Von Elm E, You J, et al. Prevalence, characteristics, and publication of discontinued randomized trials. JAMA 2014;311:1045-51.

3. Johnson CY. Chaotic search for coronavirus treatments undermines efforts, experts say. The Washington Post 2020 Apr. 15.

4. A randomised, embedded, multi-factorial, adaptive platform trial for communityacquired pneumonia (REMAP-CAP). ClinicalTrials.gov: NCT02735707; 2016. Accessible ici : www.icnarc.org/Our-Research/Studies/Remap-Cap/About (consulté le 2 déc. 2020).

5. Lamontagne F, Agoritsas $\mathrm{T}$, Macdonald $\mathrm{H}$, et al. A living WHO guideline on drugs for covid-19. BMJ 2020;370:m3379.

6. Soper B, Hanney SR. Lessons from the evaluation of the UK's NHS R\&D Implementation Methods Programme. Implement Sci 2007;2:7.

7. Evans TW. Best research for best health: a new national health research strategy. Clin Med (Lond) 2006;6:435-7.
8. Davies SC, Walley T, Smye S, et al. The NIHR at 10: transforming clinical research. Clin Med (Lond) 2016;16:501-2.

9. Atkinson P, Sheard S, Walley T. 'All the stars were aligned'? The origins of England's National Institute for Health Research. Health Res Policy Syst 2019;17:95. doi: 10.1186/s12961-019-0491-5.

10. Williams H. The NIHR Health Technology Assessment Programme: research needed by the NHS. Open Access Government [UK] 2020 Apr. 7. Accessible ici : www.openaccessgovernment.org/nihr-health-technology-assessment-programme -nhs/85065/ (consulté le 2 déc. 2020).

11. Clinical Research Network. UK: National Institute for Health Research. Accessible ici : www.nihr.ac.uk/explore-nihr/support/clinical-research-network.htm (consulté le 2 déc. 2020).

12. Iacobucci G. Clinical research adds billions to UK economy, analysis shows: BMJ 2019;367:16052.

13. Guthrie S, Hafner M, Bienkowska-Gibbs T, et al. Returns on research funded under the NIHR Health Technology Assessment (HTA) Programme: economic analysis and case studies. Rand Health Q 2016;5:5.

14. Bentley C, Cressman S, van der Hoek K, et al. Conducting clinical trials - costs, impacts, and the value of clinical trials networks: a scoping review. Clin Trials 2019;16:183-93.

15. Quebec COVID - Pandemic Network (RQCP). Quebec: Fonds de recherche du Quebec - Santé; 2020. Accessible ici : https://rqcp.ca/groupes-de-travail /\#recherche-clinique (consulté le 2 déc. 2020).

16. Collaborating Network of Networks for Evaluating COVID-19 and Therapeutic Strategies (CONNECTS). Bethesda (MD): National Heart, Lung, and Blood Institute; 2020. Accessible ici : www.nhlbi.nih.gov/science/collaborating-network-networks -evaluating-covid-19-and-therapeutic-strategies-connects (consulté le 2 déc. 2020).
Intérêts concurrents : François Lamontagne a été rémunéré par les Instituts de recherche en santé du Canada, le Fonds de recherche du Québec - Santé, le National Institute for Health Research (NIHR) et la Fondation Lotte \& John Hecht pour avoir fait des évaluations par les pairs. Il a codirigé l'essai « 65 Trial », un projet financé par le programme d'évaluation des technologies en santé du NIHR (PMID : 32049269) et coordonné par l'unité des essais cliniques de l'Intensive Care National Audit and Research Centre (ICNARC). Il est également codirecteur scientifique du Réseau Sepsis Canada, qui reçoit des fonds des IRSC, et codirecteur du Réseau québécois COVID Pandémie, qui lui est financé par le Fonds de recherche du Québec - Santé. Kathryn Rowan est directrice de l'ICNARC et de son unité d'essais cliniques, et également professeure honoraire à la London School of Hygiene and
Tropical Medicine. Elle déclare avoir reçu des subventions des programmes de financement de la recherche du NIHR. Aucun autre intérêt concurrent n'a été déclaré.

Cet article a été révisé par des pairs.

Affiliations : Université de Sherbrooke (Lamontagne); Centre de recherche du $\mathrm{CHU}$ de Sherbrooke (Lamontagne), Sherbrooke, Qc; Intensive Care National Audit and Research Centre (Rowan, Guyatt), Londres, Royaume-Uni; Département des méthodes, des données et de l'incidence de la recherche en santé (Guyatt), Université McMaster, Hamilton, Ont.

Collaborateurs : François Lamontagne a contribué à l'élaboration et à la conception de l'étude et a rédigé le manuscrit. Tous les auteurs ont révisé de façon critique le contenu intellectuel important du manuscrit; ils ont donné leur approbation finale pour la version destinée à être publiée et assument l'entière responsabilité de tous les aspects du travail.

Propriété intellectuelle du contenu : Il s'agit d'un article en libre accès distribué conformément aux modalités de la licence Creative Commons Attributions (CC BY-NC-ND 4.0), qui permet l'utilisation, la diffusion et la reproduction dans tout médium à la condition que la publication originale soit adéquatement citée, que l'utilisation se fasse à des fins non commerciales (c.-à-d. recherche ou formation) et qu'aucune modification ni adaptation n'y soit apportée. Pour plus de renseignements, consulter le https://creativecommons.org/ licenses/by-nc-nd/4.0/deed.fr.

Correspondance : François Lamontagne, francois.lamontagne@usherbrooke.ca 